\title{
Multibeam Echosounder Simulator Applying Noise Generator for the Purpose of Sea Bottom Visualisation
}

\author{
Wojciech Maleika, Michał Pałczyński, and Dariusz Frejlichowski \\ West Pomeranian University of Technology, Szczecin, \\ Faculty of Computer Science and Information Technology, \\ Zolnierska 49, 71-210, Szczecin, Poland \\ \{wmaleika,mpalczynski, dfrejlichowski\}@wi.zut.edu.pl
}

\begin{abstract}
Hydroacoustic data form almost the only basis for seabed imaging [1. The accuracy of scanning devices is therefore crucial for the reliability of the images. In the paper, the precision of the Simrad EM3000 multibeam echosounder was examined by means of statistical methods on real data. The maximum and mean errors were estimated, the correlation of error value and angle between the beam and the vertical line could be observed as well. The results of the experiments will help to increase the accuracy of terrain modeling of the sea bottom what will result in more realistic visualizations. The results of those experiments were implemented in the multibeam echosounder simulator.
\end{abstract}

Keywords: bathymetric data processing, multibeam echosounder, error of measurement, simulation.

\section{Introduction}

More than $70 \%$ of the Earth's surface is covered by open water. Human life and progress are both strongly connected with it. The analysis of the bottom of the sea or river has become crucial when it comes to safe sailing. Modern imagery techniques significantly enhance efficiency and safety. They are often intended for the representation of the hydroacoustic data, collected by means of SONAR technology, for the analysis of the underwater biological and physical characteristics. Among its important applications is the mapping of the shape and type of marine environments, e.g. shape and bottom type. In order to perform this task more precise methods for scanning, monitoring, and measuring are needed. Nowadays, sonar survey techniques are considered as having more advantages as opposed to traditional methods, because of their rapid and high-resolution work ([2]). Multibeam bathymetry allows to present the seabottom in a convenient and reliable way (see Fig. 1). Usually, the bathymetric data are processed by means of algorithms belonging to various disciplines, e.g. digital signal and image analysis and processing. The Digital Terrain Model (DTM) constitutes their final result. 
a)

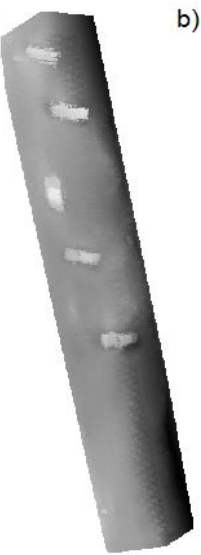

b)
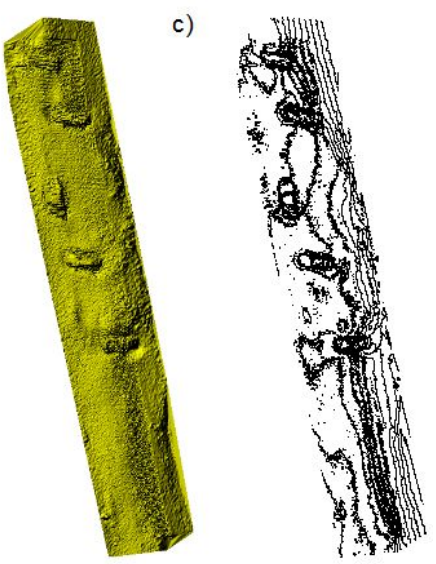

d)

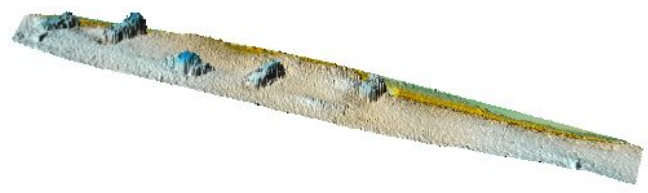

Fig. 1. Examples of DTM (Digital Terrain Model) imagery techniques (cars on the bottom of a river): a) image, b) shaded relief, c) contour map, d) 3D surface

One of the crucial problems encountered in the synthesis of the DTM based on the bathymetric data is the uncertainty of measurement, since an error in measurement may be caused by various factors. This can significantly influence the resultant image representation of the DTM. Although the measurement precision of particular devices is provided by producers, the observed errors are often higher than reference values given by manufacturers.

The problem of measurement uncertainty in case of the bathymetric data is not a new one in scientific literature. In [3] the influence of the including strong uncorrected angular variations in some tracks was stressed. In [2] the variations in the penetration of acoustic beams across different sediment types have been pointed out as the one of the five factors that hamper the proper analysis of the sediment porosity. In [4] the importance of the problem was emphasized, as according to the IHO (International Hydrographic Office) standards a few millimetres accuracy is requested. However, it is often unattainable. In some cases the error can exceed 1 meter (4]). In [5] and [6] examples of measured uncertainty have also been provided.

The problem of error distribution is very important in the field of echosounder simulation algorithms. The application of noise typical for the measurement device, or even for a specific model, increases the reliability of the generated data.

In the paper, a continuation of research described in [7] is presented, where the authors introduced the algorithm for error estimation in real sonar data. 
Here, the distribution of error is investigated. The results of noise application in echosounder simulator are presented as well.

The central problem of this work is the lack of the reference data for error estimation since there are no other sources of information about the seabed.

\section{Multibeam Echosounder and Its Simulation}

The multibeam echosounder is currently one of the most frequently applied sources of bathymetric data [1. Its most important parameters are the number of beams and beam angle. The bathymetry, even for small areas results in very large data sets, exceeding millions of points, recorded in the order of acquisition. The principles governing the multibeam echosounder operation cause the points to form lines, which are named here the measurement lines, orthogonal to the vessel's trajectory. An example of such data is shown on Fig. 2 .

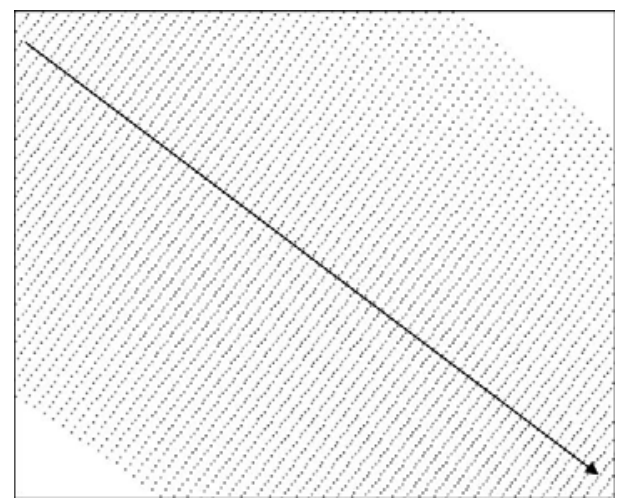

Fig. 2. The orthogonal projection of a set of bathymetric data. Measurement lines typical for multibeam echosounder can be observed. The arrow shows the ship's trajectory.

Distribution and density of bathymetric data makes them completely useless for visualization. Usually, the digital terrain model (DTM) in the form of GRID or TIN is made by means of one of the interpolation algorithms ([1]). The problem of DTM verification has driven the authors of the paper to develop the multibeam echosounder simulator, as the tool for the DTM creation research ([]). The algorithm of the simulation based on ray-tracing technique was presented in 9 . The simulation would be unreliable if it did not involve the noise generator, but the distribution of measurement errors made by real devices is unknown.

\section{Research Procedure}

Points, collected in the bathymetric data set, were grouped into consequent measurement lines, which were processed one by one. The basic concept underlying 
data accuracy investigations in this work was introduced in [7]. The research data set was collected in the area of almost flat bottom and the 10-th degree polynomial approximation of each measurement line was chosen as the reference data for error estimation.

For each point of the measurement line, the difference of depth between the profile and the point was the estimator of the measurement error. Fig. 3 presents examples of measurement lines and corresponding theoretical bottom profiles. Estimated error values were additionally classified depending on the angle between a beam and the vertical line (named here "beam angle") and error value. Angles between -90 and 90 degrees were grouped into intervals of 10 degree width, error values between 0 and $20 \mathrm{~cm}$ were grouped into intervals of $1 \mathrm{~cm}$ width. The algorithm of the data analysis is shown on Fig. 4.
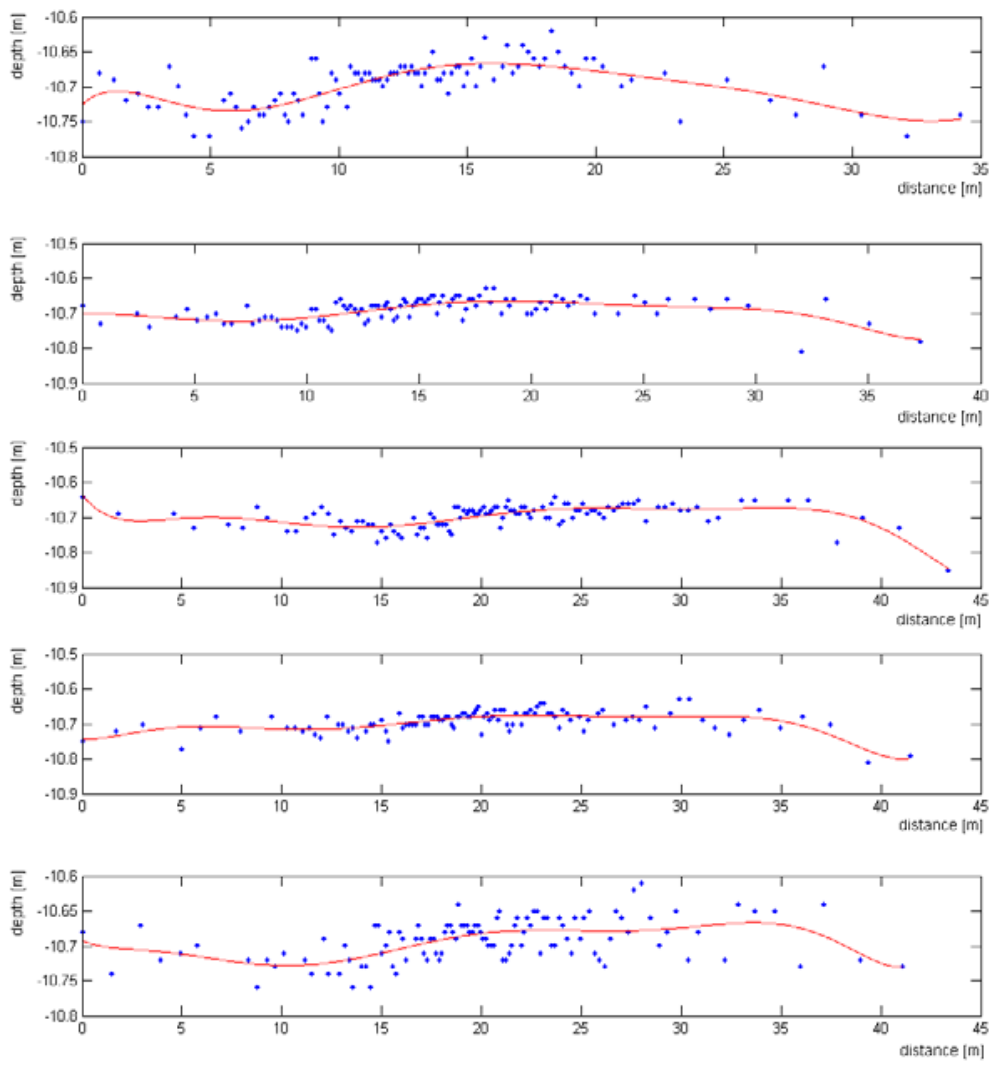

Fig. 3. Examples of measurement lines and corresponding theoretical bottom profiles 


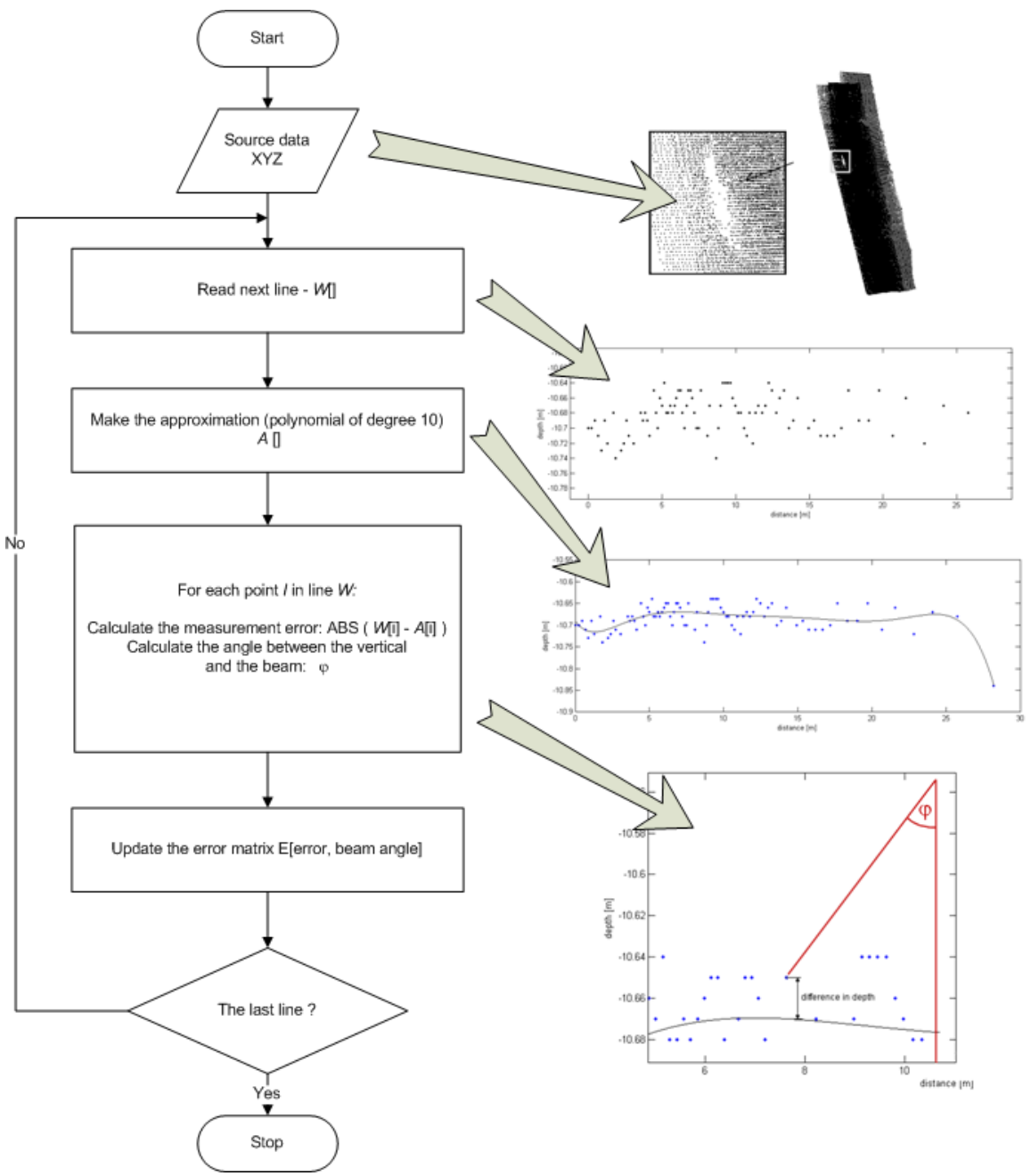

Fig. 4. The applied algorithm of the data analysis

\section{Results}

The data for the research was collected by means of the multibeam echosounder Simrad EM3000 from part of the Pomeranian Gulf (Baltic Sea). The surveyed area had the shape of a rectangle of the size $1500 \times 1400 \mathrm{~m}$, and had an almost flat bottom surface the depth of which was approximately $10 \mathrm{~m}$. The data set consisted of approx. 17 millions of points in 156 thousands of measurement lines. By means of the algorithm shown in the previous section the measurement errors were estimated, for each interval of angles separately. The quantities of errors of different values were also calculated. The analysis of the results leads 
to consideration of nearly random distribution of measurement errors in each measurement line. The dependence of the error quantities on error values seems to follow Gaussian distribution. Number of measurements a few centimeters deeper and shallower than the theoretical bottom profile was very similar, which is not dependent on the angle of beam. The distribution of error values depending on the angle of beam is presented in Fig. 5 .

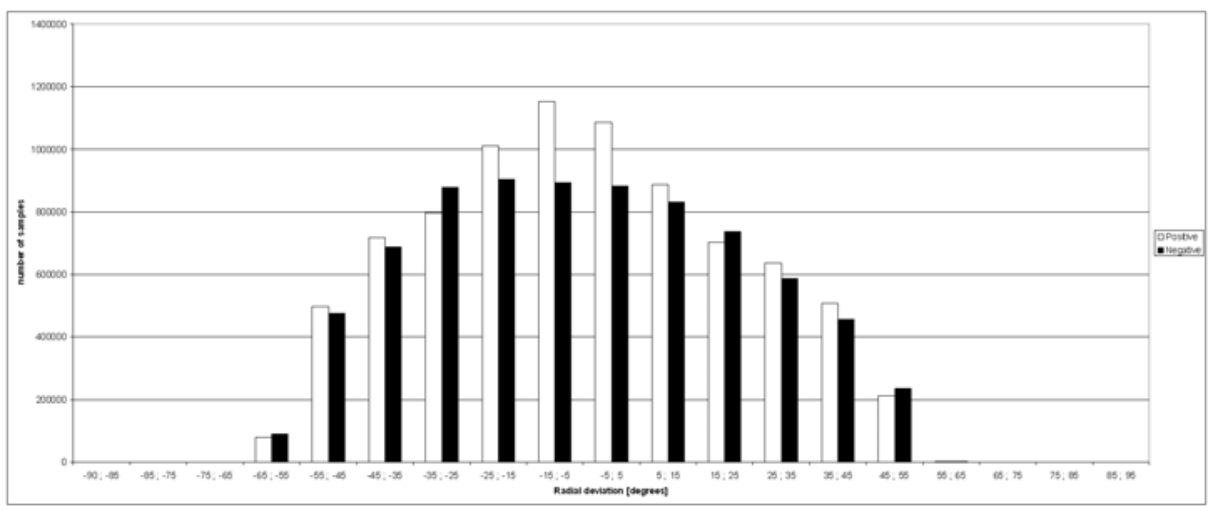

Fig. 5. The number of measurements deeper and shallower than the theoretical bottom profile for each beam angle

The average error value for all measurements was $2.25 \mathrm{~cm}(0.22 \%$ of the average depth). For $95 \%$ measurements the errors fell below $5 \mathrm{~cm}$ and for $99.7 \%$ measurements they did not exceed $7 \mathrm{~cm}$. The mean square error was equal to $3.12 \mathrm{~cm}$. The magnitudes of errors slightly increased with the beam angle. For measurements of beam angles $0-5$ degrees, the mean error was $1.9 \mathrm{~cm}$, while in case of the biggest angles (from 55 to 65 degrees), the mean error was $3 \mathrm{~cm}$ (over $50 \%$ increase). The distribution of mean error magnitudes depending on the beam angle is presented in Fig. 6.

The standard deviation was also calculated for each beam angle interval (Fig. 7). Its value increases with the beam angle (from $0.1 \mathrm{~cm}$ for the vertical measurements to $0.42 \mathrm{~cm}$ for the biggest angles). The results fulfill the natural assumption about decrease of measurement accuracy witch increase of beam angle.

\section{Application of Obtained Results in the Echosounder Simulator}

The results of experiments helped to improve the multibeam echosounder simulator by implementing a noise generator simulating real distribution of measurement errors. Next, the virtual survey was done over DTM prepared using real data. Examples of virtual measurement lines are shown on Fig. 8 . 


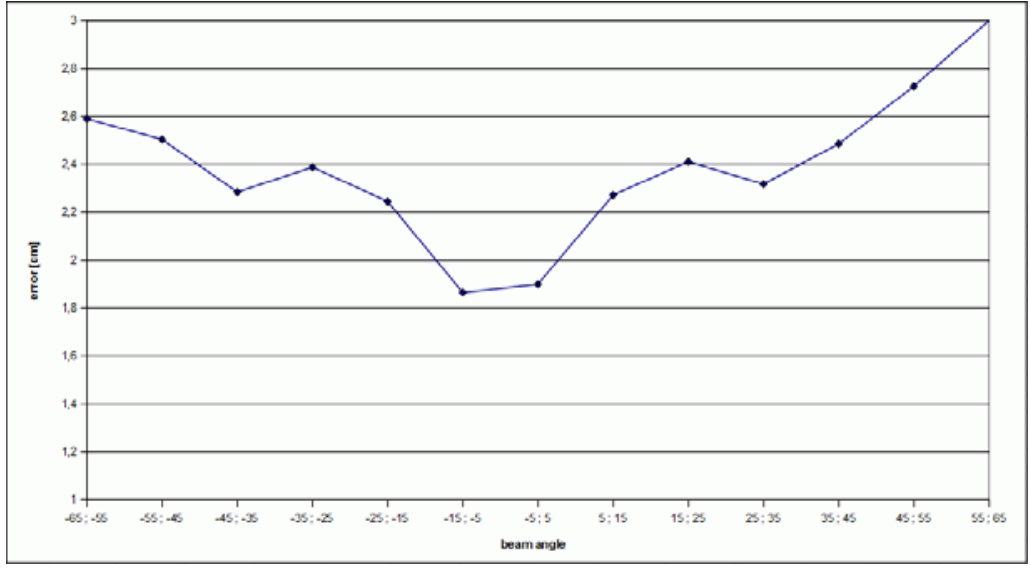

Fig. 6. Magnitudes of average errors for each beam angle interval

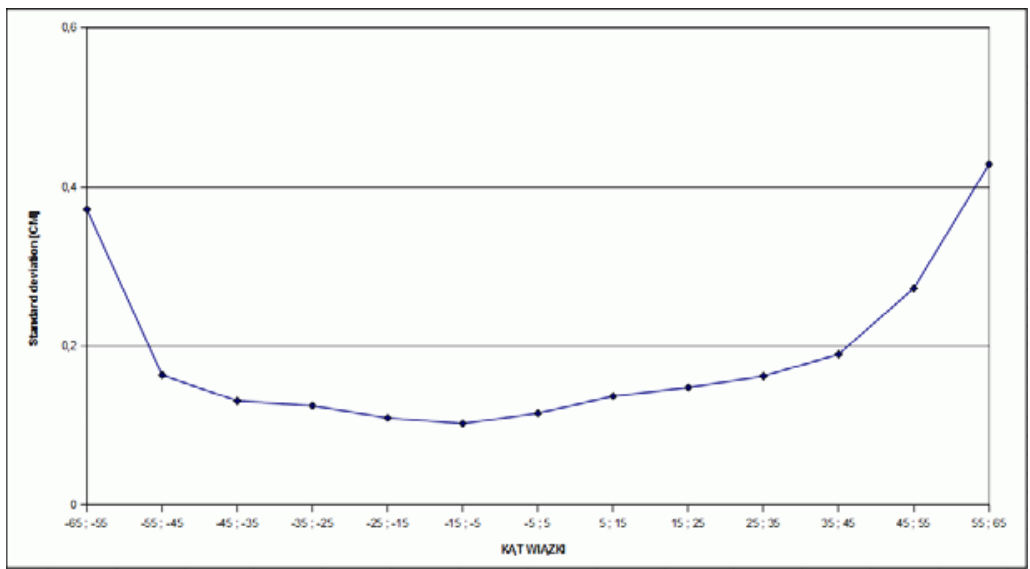

Fig. 7. Standard deviations for each beam angle interval
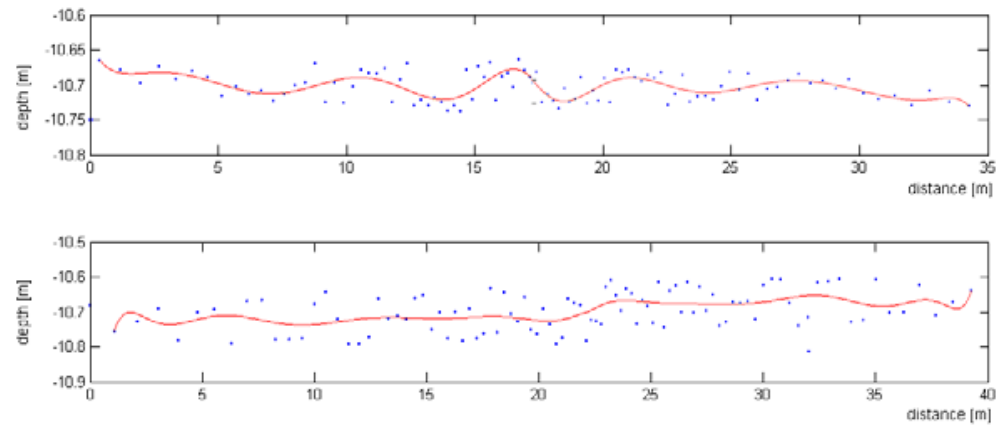

Fig. 8. Examples of virtual measurement lines synthesized using the noise generator based on the research results 
Another virtual survey was done without and with noise generating and two DTMs were created basing on both data sets. The difference between the bottom images of those models can be observed on Fig. 9 .

a)

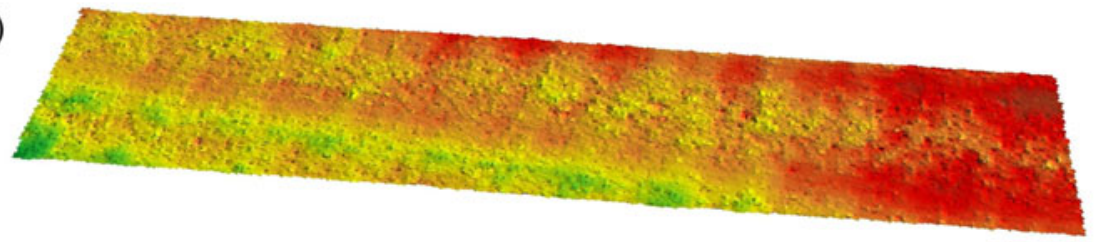

b)

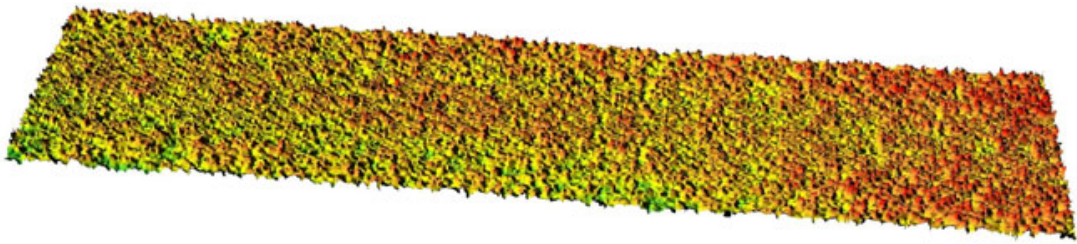

Fig. 9. 3D surfaces of digital terrain models created basing on virtual survey data a) noise generator off, b) noise generator on

\section{Conclusions}

The analysis of the data collected using the Simrad EM3000 multibeam echosounder has shown that the measurement errors are random and increase with the beam angle. The average error value $3 \mathrm{~cm}$ does not significantly differ from the error declared by the producer $(\mathrm{RMS}=5 \mathrm{~cm}[10])$. However, it should be noted that the measurements were performed on small depths. The analysis of data has shown a $50 \%$ increase of error magnitudes for measurements of the same line, depending on the beam angle.

The distribution of measurement errors estimated in the research was implemented in the noise generator of the multibeam echosounder simulator resulting in the improvement of the accuracy of DTM verification algorithms.

In the future, similar analysis of various measurement devices made by different producers could be performed. The impact of depth on the measurement error should be examined.

Acknowledgment. This work has been supported by the Ministry of Science and Higher Education of the Republic of Poland under grant no. N N526 073038.

\section{References}

1. Stateczny, A. (ed.): The methods of the comparative navigation. Scientific Association of Gdansk (2004) (in Polish)

2. Sutherland, T.F., Galloway, J., Loschiavo, R., Levings, C.D., Hare, R.: Calibration techniques and sampling resolution requirements for groundtruthing multibeam acoustic backscatter (EM3000) and QTC VIEW(TM) classification technology. Estuarine, Coastal and Shelf Science 75(4), 447-458 (2007) 
3. Blondel, P., Gomez Sichi, O.: Textural analyses of multibeam sonar imagery from Stanton Banks, Northern Ireland continental shelf. Applied Acoustics 70(10), 12881297 (2009)

4. Le Bas, T.P., Huvenne, V.A.I.: Acquisition and processing of backscatter data for habitat mapping - Comparison of multibeam and sidescan systems. Applied Acoustics 70(10), 1248-1257 (2009)

5. Brown, C.J., Blondel, P.D.: Developments in the application of multibeam sonar backscatter for seafloor habitat mapping. Applied Acoustics 70(10), 1242-1247 (2009)

6. Ferrini, V.L., Flood, R.D.: A comparison of Rippled Scour Depressions identified with multibeam sonar: Evidence of sediment transport in inner shelf environments. Continental Shelf Research 25(16), 1979-1995 (2005)

7. Maleika, W., Pałczyński, M., Frejlichowski, D., Stateczny, A.: Analysis of survey data collected using Simrad EM3000 multibeam echosounder. Methods of Applied Computer Science (4/2010), 55-64 (2010) (in Polish)

8. Maleika, W., Pałczyński, M.: Virtual multibeam echosounder in investigations on sea bottom modeling. Methods of Applied Computer Science (4/2008), 111-120 (2008)

9. Maleika, W., Pałczyński, M.: Development of Virtual Multibeam Echosounder. Accepted for Publication in WAT Bulletin, vol. 1 (2011) (in Polish)

10. http://www.kongsberg-simrad.de/pdf/faecherlot_em3000_eng_broch.pdf 